\title{
Over-expressing Akt in T cells to resist tumor immunosuppression and increase anti-tumor activity
}

\author{
Yanhong Wu, Zhenling Deng, Yishu Tang, Shuren Zhang ${ }^{*}$ and Yu-Qian Zhang*
}

\begin{abstract}
Background: Tumor employs various means to escape immunosurveillance and inhibit immune attack, and strategies have been developed to counteract the inhibitory signals. However, due to the complex suppressive mechanisms in the tumor microenvironment, blocking one or a few inhibitory signals has only limited effects on therapeutic efficacy. Instead of targeting tumor immunosuppression, we considered from another point of view, and hypothesized that manipulating $T$ cells to make them resist any known or unknown suppressive mechanism may be more effective for cancer treatment.
\end{abstract}

Methods: We used OT-1 cells transduced with retroviruses encoding Akt and human peripheral blood lymphocytes (PBLs) transduced with retroviruses encoding both Akt and a chimeric antigen receptor (CAR) specific for tumor antigen EpCAM to examine the effect of over-expressing Akt on tumor specific T cells in tumor environment.

Results: We show that Akt activity of T cells in the tumor environment was inhibited, and over-expressing Akt in OT-1 cells increased the cytokine production and cell proliferation in the presence of B16-OVA tumor cells. What's more, adoptive transfer of OT-1 cells over-expressing Akt inhibited B16-OVA tumor growth and prolonged mouse survival. To examine if over-expressing Akt could increase the anti-tumor activity of $T$ cells in human cancer, PBLs co-expressing EpCAM specific CAR and Akt were cultured with EpCAM-expressing human prostate cancer cells PC3M, and less inhibition on cell proliferation and less apoptosis were observed. In addition, adoptive transfer of PC3M specific T cells over-expressing Akt resulted in more dramatic tumor inhibitory effects in PC3M bearing NOD/SCID mice.

Conclusions: These data indicates that over-expressing Akt in tumor specific T cells increases T cell proliferation and activity in the tumor environment, and enhances anti-tumor effects of adoptively transferred T cells. Our study provides a new strategy to improve the efficacy of adoptive T cell therapy, and serves as an important foundation for clinical translation.

\section{Background}

Tumor immunosuppressive microenvironment is the major obstacle for successful clinical translation of immunotherapeutic approaches. Tumor employs different strategies to escape immunosurveillance, including impairment of the antigen presentation, up-regulating negative costimulatory signals, secretion of immunosuppressive factors, activation of pro-apoptotic pathways, and recruitment of different regulatory cell populations $[1,2]$. By these

\footnotetext{
*Correspondence: zhangsr@cicams.ac.cn; yqzhang.cams@gmail.com Department of Immunology, Cancer Hospital \& Institute, Peking Union Medical College and Chinese Academy of Medical Sciences, Beijing 100021,
} China

\section{Biomed Central}

various means, tumor induces a complex immunosuppressive microenvironment to evade immune response and restrict the effectiveness of cancer vaccine and adoptive transfer of tumor specific T cells.

With deeper understanding of the interactions between tumor and immune system, therapeutic strategies have been developed to resist immunosuppression, such as using antibodies to block CTLA-4 or PD-1 signaling, inhibiting IDO activity, depleting regulatory $\mathrm{T}$ cells, etc. [3]. However, it's easy to understand that, confronting such a complex immunosuppressive microenvironment, strategies targeting one or two inhibitory signals have only limited effects on therapeutic efficacy. Instead of dealing with 
multiple inhibitory factors, we considered if there is any means to manipulate effector $\mathrm{T}$ cells to make them resist any known or unknown immunosuppressive mechanism. Through analysis of $\mathrm{T}$ cell signaling pathways, we found that Akt is in the central node of immune modulation.

The serine/threonine kinase Akt (PKB) is utilized in a variety of signaling pathways from $\mathrm{T}$ cell growth factors such as IL-7R, and CD28 co-stimulatory signal $[4,5]$. CD28 activation enables recruitment and activation of phosphatidylinositol 3-kinase (PI3K), resulting in the generation of phosphatidylinositol-3,4,5-trisphosphate (PIP3), which recruits pleckstrin homology (PH) domain containing proteins including Akt to the plasma membrane. After recruitment to the plasma membrane, Akt becomes phosphorylated and activated by PDK1, and then plays an important role in diverse cellular processes including cell survival, glucose metabolism, and cytokine synthesis [6-8]. Besides co-stimulatory receptors, coinhibitory receptors also regulate Akt activation. Ligation of CTLA-4 and PD-1 both inhibit Akt activity, suggesting PI3K-Akt signaling is a major mechanism of immune regulation $[9,10]$. Consistent with this, it has been reported that $\mathrm{T}$ cells expressing constitutively active Akt displayed increased viability in the absence of stimulation, and could grow rapidly and secrete cytokines in the absence of CD28 co-stimulation [11].

Based on these findings, we hypothesize that upregulating Akt activity in tumor specific $\mathrm{T}$ cells could help $\mathrm{T}$ cells resist tumor immunosuppression and improve the anti-tumor effects of adoptive immunotherapy. To test this hypothesis, we used two different tumor models, B16-OVA tumor model and human prostate cancer PC3M tumor model, and demonstrated that over-expressing Akt in tumor specific T cells could increase $\mathrm{T}$ cell proliferation and cytokine secretion when co-cultured with tumor cells, and inhibit tumor growth in vivo. Our data suggests over-expressing Akt in effector T cells as a new strategy to improve the efficacy of adoptive $\mathrm{T}$ cell transfer for cancer.

\section{Methods}

\section{Mice}

OT-1 transgenic mice were purchased from the Jackson Laboratory, C57BL/6 mice and NOD/SCID mice were purchased from Vital River Laboratories. All mice were maintained under specific pathogen-free conditions in the animal facility of Cancer Institute, Chinese Academy of Medical Sciences (CAMS), and all procedures for animal experiments were approved by the Institutional Animal Use and Care Committee of CAMS.

\section{Cell lines}

The OVA-expressing tumor cell line, B16-OVA, was kindly provided by T.-C. Wu lab (Johns Hopkins
Medical Institutions, Baltimore, MD) [12], and cultured in RPMI1640 supplemented with $10 \%$ FBS, 2 mM Lglutamine, $1 \mathrm{mM}$ sodium pyruvate, $2 \mathrm{mM}$ non-essential amino acids, 50 units $/ \mathrm{ml}$ penicillin and streptomycin. PC3M cell line was purchased from ATCC, and maintained in culture with DMEM medium (Gibco) supplemented with $10 \%$ FBS.

\section{Retroviral vector construction and retrovirus preparation} PLNCX-myr-HA-Akt1 (William Sellers, Addgene plasmid 9005) and pLNCX-HA-Akt1 (William Sellers, Addgene plasmid 9004) were obtained from Addgene [13]. PLNCX empty vector was used as the control retrovirus. To construct EpCAM specific CAR and Akt co-expressing construct, HindIII/HpaI/BglII-IRES-XhoI/SalI/ClaI sequence was synthesized (IRES sequence corresponds to 11191693 of pIRES-G, with $1346 \mathrm{G} \rightarrow \mathrm{C}$ ) and inserted into HindIII/ClaI multiple cloning sites of pLNCX retroviral vector to make pLNCX-IRES. EpCAM specific CAR consisting of an anti-EpCAM scFv (sequence corresponds to Genebank identifier AJ564232.1) [14], part of the extracellular domain and the entire transmembrane and intracellular domains of CD28, and the cytoplasmic domain of $\mathrm{CD} 3 \zeta$ (the $\mathrm{CD} 28$ and $\mathrm{CD} 3 \zeta$ sequence included corresponds to Genebank identifier HM852952.1) [15] was synthesized and inserted into HindIII/BgIII of pLNCXIRES to make pLNCX-CAR. Akt and myr-Akt were cloned from pLNCX-HA-Akt1 and pLNCX-myr-HAAkt1 respectively, and inserted into XhoI/ClaI of pLNCX-CAR to make pLNCX-CAR-Akt and pLNCXCAR-myr-Akt co-expressing constructs. Retroviruses were prepared using Retrovirus Packaging Kit Ampho (TaKaRa) and $293 \mathrm{~T}$ packaging cell line, following the manufacturer's instruction.

\section{Retroviral transduction of OT-1 cells and human PBLs}

Splenocytes and lymph node cells of OT-1 transgenic mice were harvested, and red blood cells were removed by $\mathrm{RBC}$ lysis buffer (Biolegend). $\mathrm{CD}^{+} \mathrm{T}$ cells were isolated using mouse $\mathrm{CD}^{+} \mathrm{a}^{+} \mathrm{T}$ cell isolation kit (Miltenyi Biotec), and then were stimulated with $1 \mathrm{ug} / \mathrm{ml}$ antimouse CD3 and $1 \mathrm{ug} / \mathrm{ml}$ anti-mouse CD28 antibodies (Biolegend) for 2 days at $1 \times 10^{6}$ cells $/ \mathrm{ml}$ in RPMI medium (Gibco) supplemented with $10 \%$ FBS, 1x nonessential amino acid, L-glutamine, sodium pyruvate, and penicillin-streptomycin, and $0.1 \% \beta$-mercaptoethanol. For retrovirus transduction, 24-well plates were coated with RetroNectin (TaKaRa) at $4{ }^{\circ} \mathrm{C}$ overnight according to the product's manual, and blocked with $2 \%$ BSA at room temperature for $30 \mathrm{~min}$. After that, the plates were added with retrovirus supernatants at $300 \mathrm{ul} /$ well and incubated at $37{ }^{\circ} \mathrm{C}$ for $6 \mathrm{~h}$. Then the stimulated cells were transferred to the pre-coated wells at $1 \times 10^{6}$ cells $/ \mathrm{ml}$ 
and cultured at $37{ }^{\circ} \mathrm{C}$ for 5 days in the presence of $100 \mathrm{U} / \mathrm{ml}$ recombinant human IL-2.

For PBL preparation, donor blood was obtained from healthy volunteers with consent from institutional review board of Cancer Institute, Chinese Academy of Medical Sciences, and written informed consent for participation in the study was obtained from participants. PBMCs were prepared by centrifugation of the blood on Ficoll-Hypaque density gradients (Sigma-Aldrich), and adherent cells were removed by plating in cell culture plates for $2 \mathrm{~h}$. Then the cells were stimulated with $1 \mathrm{ug} / \mathrm{ml}$ OKT3 and $1 \mathrm{ug} / \mathrm{ml}$ anti-human CD28 antibodies (Biolegend) at $1 \times 10^{6}$ cells $/ \mathrm{ml}$ for 2 days, and transduced with retroviruses as described above.

\section{Western blot}

Cell lysis was performed on ice for $30 \mathrm{~min}$ in RIPA buffer with $1 \mathrm{x}$ protease inhibitor cocktail, $0.5 \mathrm{mM}$ PMSF, $30 \mathrm{mM} \mathrm{NaF}, 1 \mathrm{mM} \mathrm{Na} \mathrm{VO}_{4}$ added freshly. After centrifugation at $1000 \mathrm{rpm}$ at $4{ }^{\circ} \mathrm{C}$ for $10 \mathrm{~min}$, the protein concentration of the supernatant was determined by BCA assay, and 40 ug of protein extract was subjected to SDS-PAGE and western blot following standard procedures. Phospho-Akt (Ser473) Rabbit mAb (Cell Signaling Technology), Akt (pan) Rabbit mAb (Cell Signaling Technology), and $\beta$-actin antibody (Santa Cruz Biotechnology) were used at 1:2000 to probe the western blots.

\section{RT-PCR and quantitative real-time PCR}

Total RNA was extracted using the RNeasy Mini kit (Qiagen) and was reverse transcribed using ReverTra Ace qPCR RT Master Mix with gDNA Remover (TOYOBO). PCR was performed with EmeraldAmp MAX PCR Master Mix (TAKARA), and the following primers were used: CAR, $5^{\prime}$ - CCTTATGGTTACGACGAGTAT GGTCTGG and 3'- AACAGTTTAGGAGGCTGCCCTG GTTTCT; $\beta$-actin, 5'-AAGAGAGGCATCCTCACCCT and 3' - TACATGGCTGGGGTGTTGAA. Real-time PCR was performed with iTaq Universal SYBR Green Supermix (BIO-RAD) using CFX96 Touch Real-Time PCR Detection System (BIO-RAD), and the primers were purchased from GeneCopoeia (Catalog\# HQPCS0002).

\section{Flow cytometry}

Cells were stained with FITC or PE-conjugated CD8, IFN- $\gamma$, HLA-DR, CD80, B7-H1, TGF- $\beta$, IL-10 antibodies following standard protocol. All antibodies were purchased from Biolegend. CFSE cell proliferation kit (Life Technologies) was used to determine cell proliferation, and APC Annexin V apoptosis detection kit with PI (Biolegend) was used to detect cell apoptosis. Cells were analyzed by a FACS Calibur flow cytometer and Flowjo software.

\section{Luminescence imaging}

Luciferase expressing cell line PC3M-luc was established by transducing PC3M with retroviruses encoding luciferase and selection with G418. To examine the cytotoxicity of PBLs transduced with different retroviruses, PC3M-luc cells were incubated with effector cells for $24 \mathrm{~h}$, and then luciferin (Promega) was added at a final concentration of $0.3 \mathrm{mg} / \mathrm{ml} .10 \mathrm{~min}$ later, luminescence imaging was conducted on IVIS system (Xenogen/Caliper Life Sciences).

\section{Statistical analysis}

Data are presented as mean \pm SE. To determine the significance of differences between samples or groups, the student's $t$-test or two-way ANOVA was used as indicated in the figure legends.

\section{Results}

Akt expression in the T cells is down-regulated in the tumor microenvironment

To verify that Akt activation in $\mathrm{T}$ cells is inhibited in tumor environment, $\mathrm{T}$ cells were isolated from OT-1 transgenic mice, stimulated with anti-CD3 and antiCD28 antibodies, and then incubated with B16-OVA tumor culture medium supernatant. $24 \mathrm{~h}$ later, the levels of phosphorylated Akt were determined by western blot. As shown in Fig. 1a, incubation with B16-OVA culture supernatant inhibited Akt phosphorylation, suggesting that Akt activation in $\mathrm{T}$ cells is inhibited by tumor secreted factors. To examine Akt activation state of T cells in tumor microenvironment in vivo, T cells were isolated from tumor draining lymph nodes (TDLN) and distant lymph nodes (LN) of B16-OVA tumor bearing mice, and Akt phosphorylation was detected. As we expected, the phosphorylation of Akt was down-regulated in TDLN comparing to distant LN (Fig. 1b).

\section{Over-expressing Akt in OT-1 cells enhances anti-tumor effect in mouse B16 melanoma model}

To examine if up-regulating Akt activation could increase the activity of T cells in the presence of tumor, we transduced OT-1 cells with retroviruses encoding wild type Akt (wtAkt) or a constitutively active form of Akt (myristoylated Akt, myr-Akt), and incubated the transduced OT-1 cells with B16-OVA tumor cells. As shown in Fig. 2a, when co-cultured with B16-OVA, OT-1 cells transduced with either wtAkt or myr-Akt produced more IFN- $\gamma$ than OT-1 cells transduced with control retroviruses. In the proliferation assay, OT-1 cells transduced with control retroviruses slowed proliferation on exposure to B16-OVA tumor cells, whereas OT-1 cells transduced with either wtAkt or myr-Akt displayed higher proliferation rates when cultured alone, and further increased the percentage of proliferative populations when 


\section{A \\ OT-1

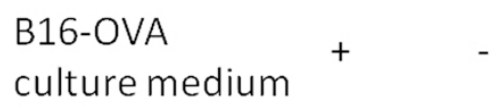

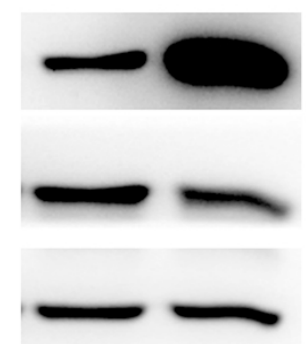

B B16-OVA tumor bearing mice

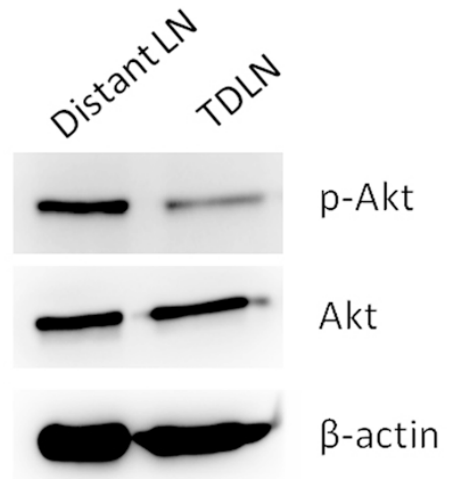

Fig. 1 Akt activity of T cells is down-regulated in tumor environment. a CD8 ${ }^{+} \mathrm{T}$ cells isolated from OT-1 transgenic mice were stimulated with anti-CD3 and anti-CD28 antibodies for 2 days, and then incubated with or without B16-OVA tumor cell culture supernatant. 24 h later, cells were collected, and the levels of Akt, phosphorylated Akt, and $\beta$-actin were examined by western blot. $\mathbf{b}$ CD $8^{+} \mathrm{T}$ cells were isolated from tumor draining lymph nodes (TDLN) or distant lymph nodes of B16-OVA tumor bearing mice, the levels of Akt, phosphorylated Akt, and $\beta$-actin were examined by western blot

co-cultured with B16-OVA (Fig. 2b). Together, these data demonstrates that over-expressing Akt or myr-Akt could increase the cytokine production and proliferation of tumor specific $\mathrm{T}$ cells in the presence of tumor.

To investigate if transducing wtAkt or myr-Akt could improve the therapeutic effect of adoptively transferred $\mathrm{T}$ cells against tumor, C57BL/6 mice were inoculated with B16-OVA tumor cells subcutaneously, when tumor size reached $\sim 6 \mathrm{~mm}$, in vitro stimulated and expanded OT-1 cells transduced with control retroviruses or retroviruses encoding wtAkt or myr-Akt were intratumorally injected into mice. As shown in Fig. 3a, adoptive transfer of control OT-1 cells didn't inhibit tumor growth comparing to the untreated group, whereas transducing wtAkt significantly increased the anti-tumor effect and prolonged mouse survival (Fig. 3b).

\section{Human T cells engineered to over-express Akt exert enhanced anti-tumor activity}

To verify the above findings and further characterize the mechanisms by which wtAkt or myr-Akt transduced $\mathrm{T}$ cells resist tumor immunosuppression, we used the human prostate cancer model we established previously in our lab [16]. Prostate cancer cell line PC3M highly expresses EpCAM, and human peripheral blood lymphocytes (PBLs) were engineered to recognize PC3M by transducing with EpCAM specific chimeric antigen receptor (CAR) which consists of an anti-EpCAM scFv, part of the extracellular domain and the entire transmembrane and intracellular domains of CD28, and the cytoplasmic domain of $\mathrm{CD} 3 \zeta$. As shown in Fig. 4a, PC3M lacks expressions of HLA-DR and CD80, whereas expresses high levels of B7-H1, TGF- $\beta$, and IL-10. To enhance the anti-tumor activity of PBLs expressing EpCAM specific CAR against the immunosuppressive PC3M tumor, we generated CAR-Akt, or CAR-myr-Akt co-expressing retroviral vectors (Fig. $4 \mathrm{~b}$ ), and verified the transduction efficiency of CAR and Akt in PBLs by RT-PCR, real-time PCR and western blot, respectively (Fig. 4c). The transduction efficiency of CAR was also examined by staining with protein $\mathrm{L}$, which is an immunoglobulin binding protein that can be used to determine CAR expression [17] (Additional file 1: Figure S1). Since it was reported that Akt inhibition promotes $\mathrm{T}$ cell differentiation to a memory phenotype [18], we evaluated CD62L expression in transduced T cells. As shown in Additional file 2: Figure S2, Akt over-expression didn't down-regulate the level of CD62L.

To determine if co-expressing Akt or myr-Akt could help PBLs resist tumor immunosuppression, PBLs transduced with CAR, CAR-Akt, or CAR-myr-Akt were cocultured with PC3M tumor cells, 3 days later, $\mathrm{T}$ cell proliferation and apoptosis were examined. As shown in Fig. 5 and Additional file 3: Figure S3, T cell proliferations were all inhibited in the presence of tumor cells, however, the degree of inhibition was less in T cells coexpressing Akt or myr-Akt. In addition, incubation with tumor cells increased cell apoptosis in all groups, however, T cells co-expressing Akt displayed less apoptosis comparing to $\mathrm{T}$ cells expressing CAR only, and $\mathrm{T}$ cells transduced with myr-Akt displayed higher apoptosis rate than $\mathrm{T}$ cells transduced with wtAkt, suggesting that transducing wtAkt may be more beneficial than myr-Akt.

Next, we examined the anti-tumor effects of PBLs coexpressing Akt or myr-Akt both in vitro and in vivo. PBLs transduced with CAR, CAR-Akt, or CAR-myr-Akt 

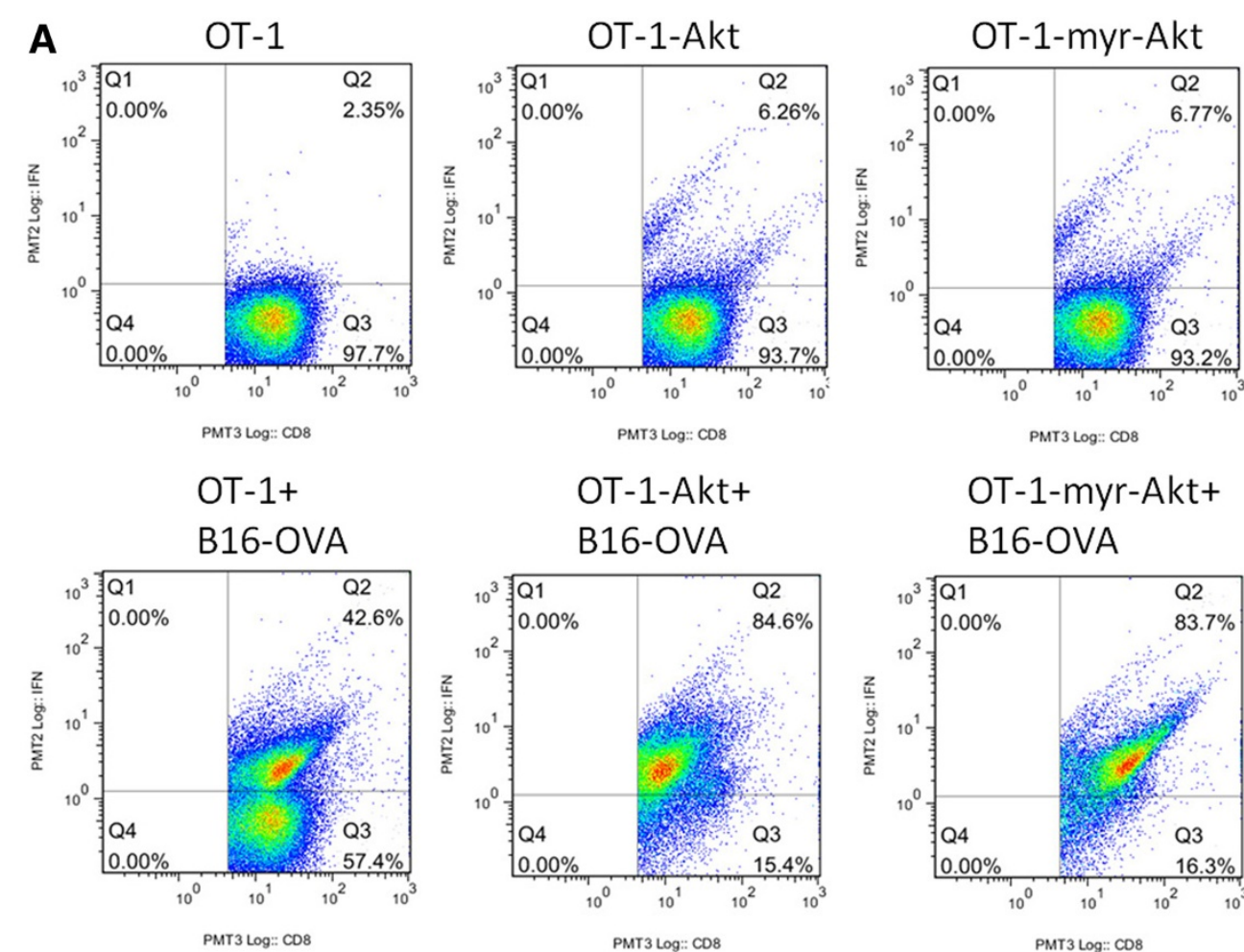

OT-1-myr-Akt+ B16-OVA

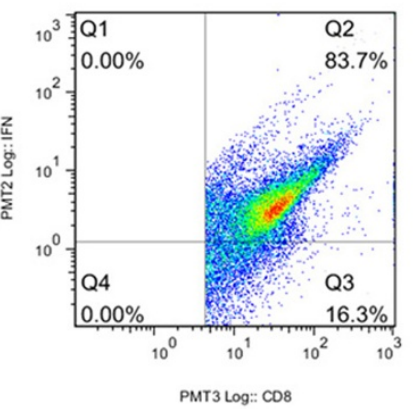

B
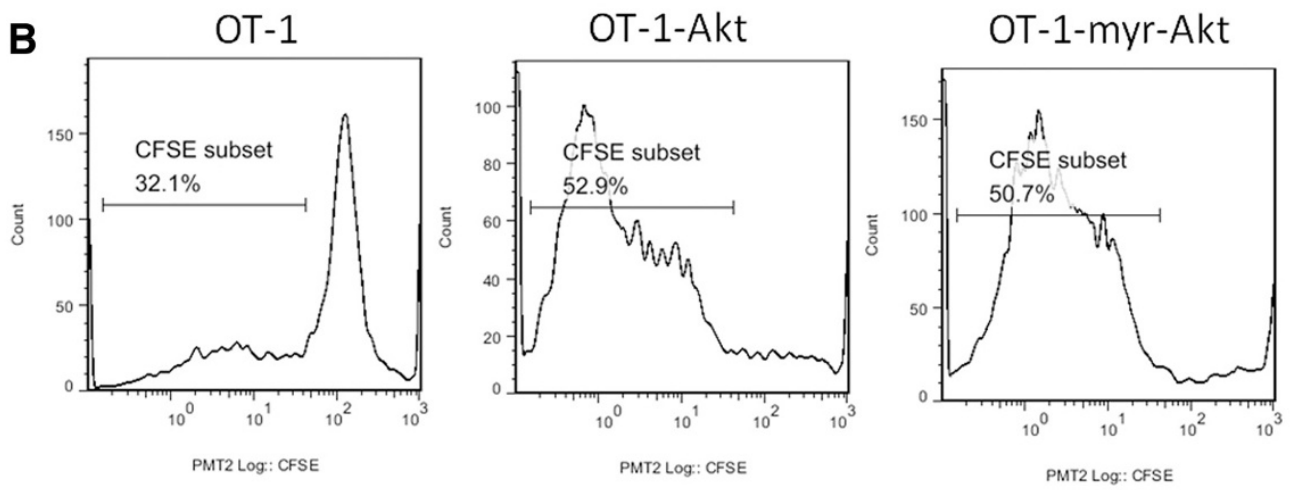

OT-1+

B16-OVA

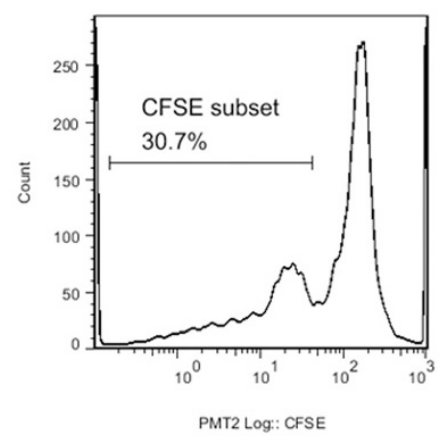

PMT2 Log: CFSE

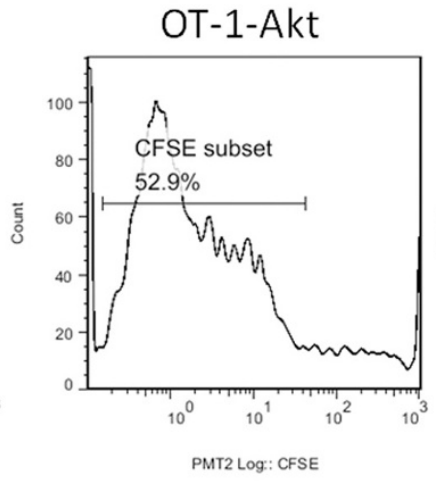

OT-1-Akt+

B16-OVA

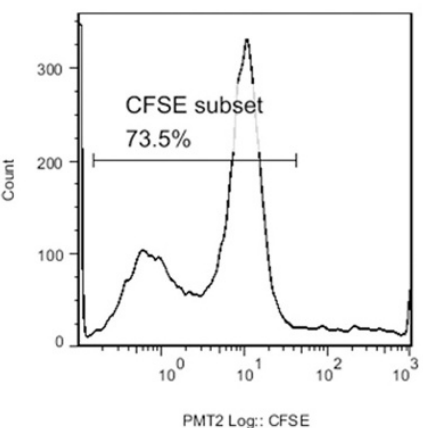

Fig. 2 (See legend on next page.) 
(See figure on previous page.)

Fig. 2 OT-1 cells transduced with Akt or myr-Akt display increased IFN- $-\gamma$ production and proliferation in the presence of B16-OVA tumor cells. a OT-1 cells transduced with control retroviruses or retroviruses encoding Akt or myr-Akt were incubated with or without B16-OVA tumor cells at an E:T ratio of 2:1, $24 \mathrm{~h}$ later, the cells were stained with anti-CD8 and anti-IFN- $\gamma$ antibodies, and analyzed by flow cytometry. The analysis was carried out with gated $\mathrm{CD} 8^{+}$population. $\mathbf{b}$ OT-1 cells transduced with control retroviruses or retroviruses encoding Akt or myr-Akt were labeled with CFSE, and co-cultured with or without B16-OVA tumor cells at an E:T ratio of 2:1, 3 days later, the cells were stained with anti-CD8 antibody, and analyzed by flow cytometry. The analysis was carried out with CD8 ${ }^{+}$population

were incubated with luciferase-expressing stable cell line PC3M-luc, $24 \mathrm{~h}$ later, cytotoxicity was determine by luminescence imaging. As shown in Fig. 6a, PBLs coexpressing Akt caused more cell lysis than PBLs expressing CAR only. In NOD/SCID mice, PC3M tumor cells were inoculated subcutaneously, when tumor size reached $\sim 3 \mathrm{~mm}$, PBLs transduced with CAR, CAR-Akt, or CAR-myr-Akt were injected intratumorally. As shown in Fig. 6b, PBLs co-expressing Akt significantly inhibited tumor growth comparing to PBLs expressing CAR only. These data indicates that Akt transduction can increase the activity of $\mathrm{T}$ cells in tumor environment and enhance the anti-tumor efficacy of adoptively transferred tumor specific $\mathrm{T}$ cells.

\section{Discussion}

In this study, we show that Akt activity of $\mathrm{T}$ cells is down-regulated in tumor environment, and transducing Akt increases the proliferation, cytokine production, and anti-tumor effects of tumor specific T cells.

Akt phosphorylations in T cells incubated with tumor culture supernatant or T cells isolated from TDLN were inhibited (Fig. 1a and b), consistent with previous findings that the activation status of Akt in T cells is a primary determinant of $\mathrm{T}$ cell sensitivity to tumor immunosuppression [19]. It's noticeable that OT-1 cells transduced with myr-Akt didn't shown further enhanced effector functions than OT-1 cells transduced with wtAkt, which was also demonstrated in PC3M tumor

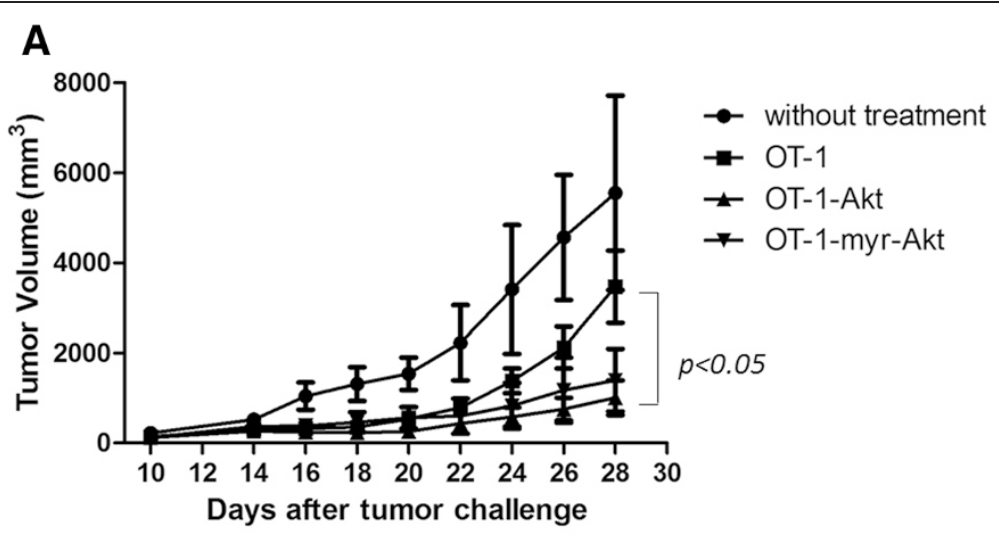

B

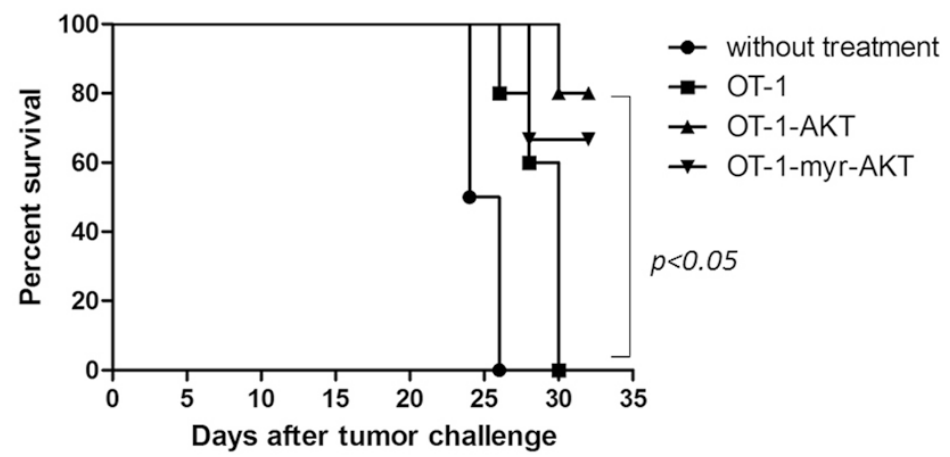

Fig. 3 Adoptive transfer of OT-1 cells transduced with Akt can inhibit B16-OVA tumor growth and prolong mouse survival. OT-1 cells transduced with control retroviruses or retroviruses encoding Akt or myr-Akt were intratumorally injected into B16-OVA tumor bearing mice at $2 \times 10^{6}$ cells/ mouse at 11 days after tumor challenge, and the mice without treatment were used as control. Tumor growth was monitored every other day, and the death of mouse was defined when the tumor size reached $2 \mathrm{~cm}$. a Line graph depicting the tumor volume of mice, statistical analysis was performed using two-way ANOVA. b Kaplan-Meier survival analysis of transduced OT-1 treated or untreated mice. Comparison of survival curves was made by Log-rank test 


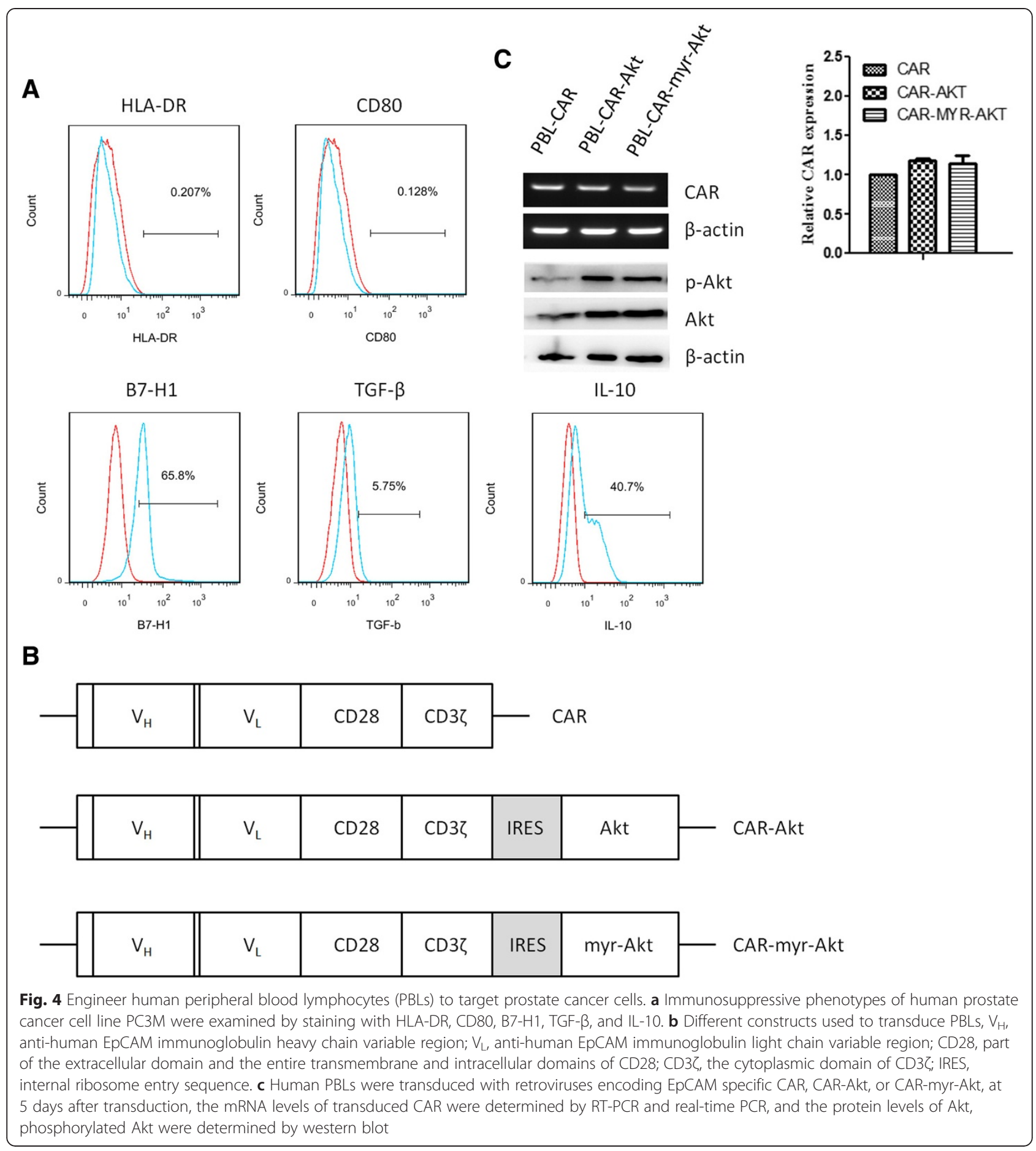

model. It could be partly explained by the similar phosphorylation levels of Akt observed for T cells transduced with wtAkt and myr-Akt (Fig. 4c), also the increased apoptosis observed for PBLs transduced with myr-Akt cultured alone or in the presence of tumor cells (Fig. 5b). The increased apoptosis observed for PBLs transduced with myr-Akt is inconsistent with previous report that $\mathrm{T}$ cells expressing constitutively active Akt up-regulated anti-apoptotic molecules and displayed decreased apoptosis [20]. This difference could be caused by the different experimental system used in their study, in which the apoptosis was evaluated at 4 weeks after retrovirus transduction, whereas our study characterized $\mathrm{T}$ cells at 5 days after transduction.

The increased apoptosis observed for myr-Akt transduced $\mathrm{T}$ cells is contradictory to the well known pro- 

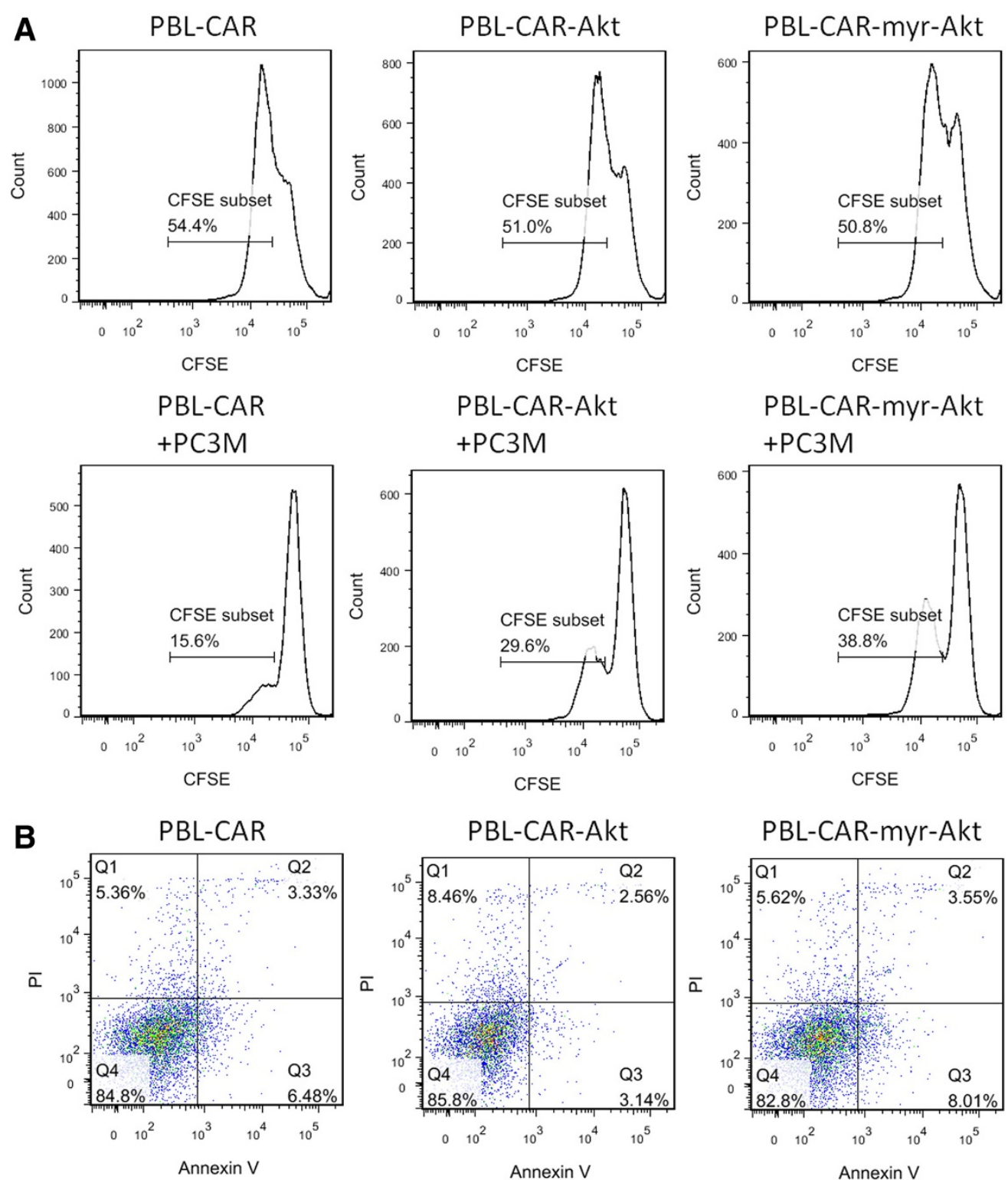

\section{PBL-CAR-myr-Akt}

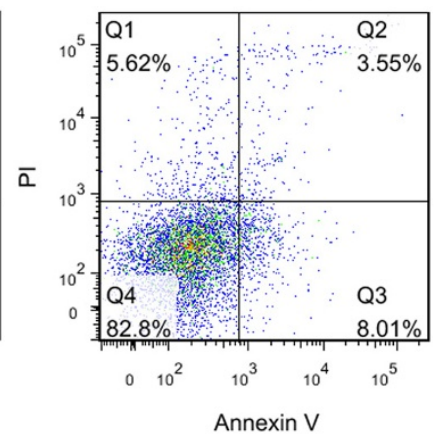

PBL-CAR

$+\mathrm{PC} 3 \mathrm{M}$
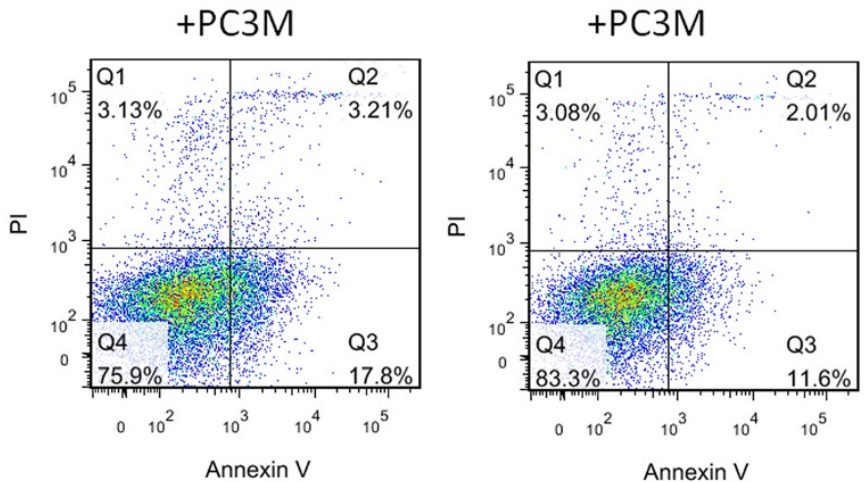

PBL-CAR-myr-Akt $+P C 3 M$

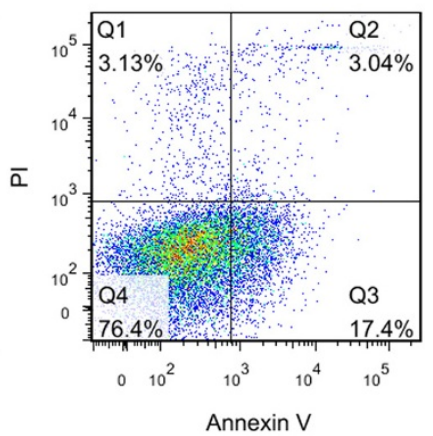

Fig. 5 Over-expressing Akt increases the activity of CAR expressing PBLs in the presence of PC3M. PBLs transduced with CAR, CAR-Akt, or CAR-myr-Akt were co-cultured with or without PC3M at an E:T ratio of 2:1, 3 days later, cell proliferation was examined by CFSE assay (a), and cell apoptosis was determined by Annexin V/PI staining (b), the analysis was performed with gated CD $8^{+}$population 

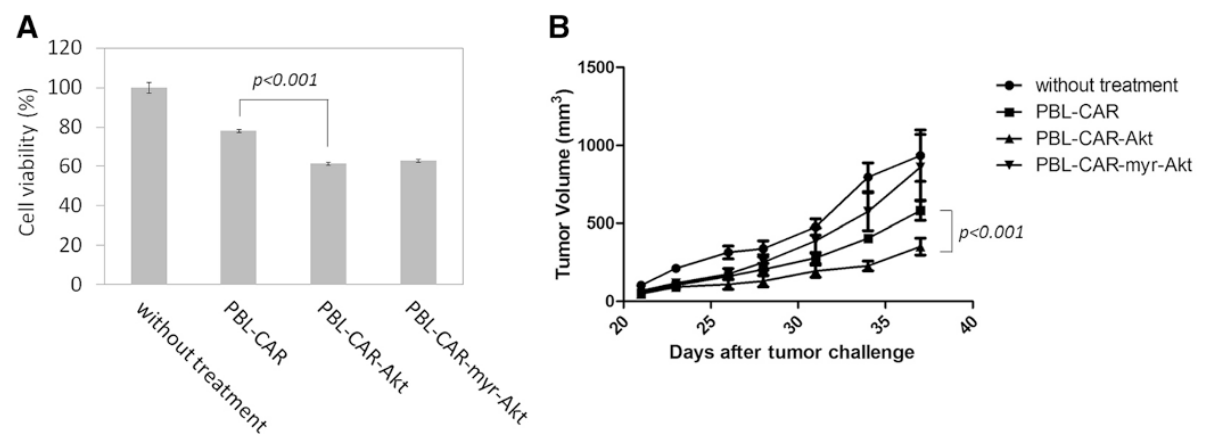

Fig. 6 Over-expressing Akt enhances the anti-tumor efficacy of PBLs in vitro and in vivo. a PBLs transduced with CAR, CAR-Akt, or CAR-myr-Akt were incubated with PC3M-luc at an E:T ratio of 2:1, $24 \mathrm{~h}$ later, cell viability was determined by luminescence imaging, and the luminescence intensity was normalized to that of PC3M-luc cells without treatment. Statistical analysis was performed using student's $t$-test. $\mathbf{b}$ NOD/SCID mice were subcutaneously injected with PC3M tumor cells at $6 \times 10^{6}$ cells/mouse, at 21 days after tumor challenge, PBLs transduced with CAR, CAR-Akt, or CAR-myr-Akt were intratumorally injected into mice at $3 \times 10^{6}$ cells/mouse, and the mice without treatment were used as control. Tumor volume was measured every few days, and statistical analysis was performed using two-way ANOVA

survival role of Akt, however, it was reported that Akt regulates Fas-mediated cell death through transcriptional activation of Fas receptor [21]. In addition, it has also been shown that Akt-dependent GSK inhibition regulates activation induced cell death (AICD) of T cells [22]. It's possible that over-expressing constitutively active Akt increased AICD of $\mathrm{T}$ cells, thus dampened the therapeutic efficacy of tumor specific $T$ cells. The fact that tumor specific $\mathrm{T}$ cells transduced with wtAkt displayed better effector functions than $\mathrm{T}$ cells transduced with myr-Akt actually makes this strategy more convenient for clinical translation. Although it has been reported that $\mathrm{T}$ cells expressing constitutively active Akt still require antigen stimulation to grow [11], transducing constitutively active Akt raises safety concerns for the application of this strategy. Our data supports overexpressing wtAkt to enhance the therapeutic effects of adoptively transferred tumor specific $\mathrm{T}$ cells, which serves as a foundation for broader clinical translation.

OT-1 cells transduced with Akt produced IFN- $\gamma$ and increased the percentage of proliferative cells on exposure to B16-OVA, indicating the specific activation of $\mathrm{T}$ cells by tumor. In contrast, PBLs expressing CAR-Akt co-cultured with PC3M displayed decreased proliferation and increased apoptosis comparing to cultured alone, suggesting that PC3M model is more immunosuppressive, and up-regulating Akt is not sufficient to enable $\mathrm{T}$ cells to completely resist the suppression. Despite this, PBLs expressing CAR-Akt increased antitumor effects both in vitro and in vivo, comparing to PBLs expressing CAR only. It's worth to mention that the EpCAM specific CAR we used consists of both $\mathrm{CD} 28$ and $\mathrm{CD} 3 \zeta$ signaling domains, thus the increased cytotoxicity displayed by PBLs co-expressing Akt is probably due to resistance to inhibitory signaling conferred by Akt activation.
The recent report by Crompton et al. found that inhibition of Akt enabled TIL a memory T cell signature with enhanced anti-tumor effect [23]. TILs isolated for adoptive transfer normally undergo extensive expansion process in vitro, and display a terminally-differentiated phenotype with short telomere and diminished antitumor activity. In their study, Akt inhibitor was added only in the expansion process, and they found that TILs expanded with Akt inhibition persisted longer and exerted better anti-tumor effects. Comparing to their study, we used OT-1 T cells or PBLs stimulated and expanded in vitro, which displayed a $\mathrm{CD} 62 \mathrm{~L}^{+}$phenotype after the short expansion process (Additional file 2: Figure S2). Akt is necessary to induce and sustain effector functions of $\mathrm{T}$ cell $[18,24]$, comparing to their study trying to enhance the persistence of $\mathrm{T}$ cells by inhibition of Akt, we tried to enhance the effector functions of $\mathrm{T}$ cells by over-expressing Akt, these two studies take different angles to manipulate $\mathrm{T}$ cells to enhance the anti-tumor efficacy, and are not contradictory to each other, and it's worth further study to determine which means is superior to the other. In addition, in their work, tumor bearing mice were pretreated with total body irradiation, adjuvant vaccine, and IL-2 in conjunction with cell therapy, these preparations could ameliorate tumor immunosuppression and enhance the anti-tumor effects of cell therapy, however, severe adverse effects have been reported. In contrast, we used adoptive cell therapy alone to treat tumor, and over-expressing Akt could enable $\mathrm{T}$ cells to resist tumor immunosuppression, which is the major advantage of our strategy.

\section{Conclusions}

In summary, our data demonstrates that over-expressing Akt in tumor specific T cells could improve the proliferation, cytokine production, and cytotoxicity of $\mathrm{T}$ cells in 
the presence of tumor. Comparing to strategies designed to counteract various immunosuppressive mechanisms employed by tumor, transducing Akt in T cells provides a new approach for tumor immunotherapy, and has important implications for improving the therapeutic effects of adoptive T cell transfer.

\section{Additional files}

Additional file 1: Figure S1. Transduction efficiency of EpCAM CAR. PBLs were transduced with CAR, CAR-Akt, or CAR-myr-Akt, 4 days later, CAR expression was detected by biotinylated Protein $L$ and PE conjugated streptavidin. A) FACS histogram B) Quantification of transduction efficiency of PBLs from 3 healthy donors. The untransduced PBLs were used as the negative control. (JPEG $82 \mathrm{~kb}$ )

Additional file 2: Figure S2. CD62 $L$ expression on transduced PBLS. PBLs were transduced with CAR, CAR-Akt, or CAR-myr-Akt, 4 days later, the cells were stained with CD4, CD8, and CD62L. A) FACS histogram B) Quantification of CD62L expression on $\mathrm{CD}^{+}$or $\mathrm{CD}^{+}$cells from 3 healthy donors. (JPEG $790 \mathrm{~kb}$ )

Additional file 3: Figure S3. Quantification of cell proliferation and apoptosis of transduced PBLs. PBLs transduced with CAR, CAR-Akt, or CAR-myr-Akt were co-cultured with or without PC3M at an E:T ratio of 2:1, 3 days later, cell proliferation and apoptosis were determined. A) Percent of CFSE ${ }^{+}$cells in CD8 ${ }^{+}$population B) Percent of Annexin $\mathrm{V}^{+}$cells in $\mathrm{CD}^{+}$population. Experiments were repeated for 3 times, and statistical analysis was performed using student's $t$-test. (JPEG $99 \mathrm{~kb}$ )

\section{Competing interests}

The authors declare that they have no competing interests.

\section{Authors' contributions}

YQZ conceived the project, designed the experiments, analyzed data, and wrote the manuscript. YW and ZD performed all experiments and analyzed data, YT contributed to the OT-1 in vitro experiments. SZ supervised experiments and helped with discussion. All authors read and approved the final manuscript.

\section{Acknowledgements}

We thank Dr. T.-C. Wu and Dr. Chien-Fu Hung for the kind gift of B16-OVA tumor cell line; Dr. William Sellers for sharing pLNCX-HA-Akt1 and pLNCXmyr-HA-Akt1 plasmids. We thank Tao Xu for assistance with flow cytometry, and Xiao Liang for assistance with luminescence imaging. This work was supported by grants from the Natural Science Foundation of China (81101554), and Chinese Academy of Medical Sciences.

Received: 9 August 2014 Accepted: 19 August 2015

Published online: 27 August 2015

\section{References}

1. Rabinovich GA, Gabrilovich D, Sotomayor EM. Immunosuppressive strategies that are mediated by tumor cells. Annu Rev Immunol. 2007;25:267-96.

2. Swann JB, Smyth MJ. Immune surveillance of tumors. J Clin Invest. 2007;117(5):1137-46.

3. Zou W. Immunosuppressive networks in the tumour environment and their therapeutic relevance. Nat Rev Cancer. 2005;5(4):263-74.

4. Pallard C, Stegmann AP, van Kleffens T, Smart F, Venkitaraman A, Spits H. Distinct roles of the phosphatidylinositol 3-kinase and STAT5 pathways in IL-7-mediated development of human thymocyte precursors. Immunity. 1999:10(5):525-35

5. Parry RV, Reif K, Smith G, Sansom DM, Hemmings BA, Ward SG. Ligation of the T cell co-stimulatory receptor CD28 activates the serine-threonine protein kinase protein kinase B. Eur J Immunol. 1997;27(10):2495-501.

6. Cantrell D. Protein kinase B (Akt) regulation and function in T lymphocytes. Semin Immunol. 2002;14(1):19-26.

7. Scheid MP, Woodgett JR. PKB/AKT: functional insights from genetic models. Nat Rev Mol Cell Biol. 2001;2(10):760-8.
8. Wu LX, La Rose J, Chen L, Neale C, Mak T, Okkenhaug K, et al. CD28 regulates the translation of $\mathrm{BCl}-\mathrm{xL}$ via the phosphatidylinositol 3-kinase/mammalian target of rapamycin pathway. J Immunol (Baltimore, Md : 1950). 2005;174(1):180-94.

9. Parry RV, Chemnitz JM, Frauwirth KA, Lanfranco AR, Braunstein I, Kobayashi SV, et al. CTLA-4 and PD-1 receptors inhibit T-cell activation by distinct mechanisms. Mol Cell Biol. 2005;25(21):9543-53.

10. Parry RV, Riley JL, Ward SG. Signalling to suit function: tailoring phosphoinositide 3-kinase during T-cell activation. Trends Immunol. 2007;28(4):161-8.

11. Rathmell JC, Elstrom RL, Cinalli RM, Thompson CB. Activated Akt promotes increased resting T cell size, CD28-independent T cell growth, and development of autoimmunity and lymphoma. Eur J Immunol. 2003;33(8):2223-32.

12. Kang TH, Lee JH, Song CK, Han HD, Shin BC, Pai SI, et al. Epigallocatechin-3gallate enhances CD8+ T cell-mediated antitumor immunity induced by DNA vaccination. Cancer Res. 2007;67(2):802-11.

13. Ramaswamy S, Nakamura N, Vazquez F, Batt DB, Perera S, Roberts TM, et al. Regulation of G1 progression by the PTEN tumor suppressor protein is linked to inhibition of the phosphatidylinositol 3-kinase/Akt pathway. Proc Natl Acad Sci U S A. 1999;96(5):2110-5.

14. Grosse-Hovest L, Hartlapp I, Marwan W, Brem G, Rammensee HG, Jung G. A recombinant bispecific single-chain antibody induces targeted, supra-agonistic CD28-stimulation and tumor cell killing. Eur J Immunol. 2003;33(5):1334-40.

15. Kochenderfer JN, Feldman SA, Zhao Y, Xu H, Black MA, Morgan RA, et al. Construction and preclinical evaluation of an anti-CD19 chimeric antigen receptor. J Immunother (Hagerstown, Md : 1997). 2009;32(7):689-702.

16. Deng Z, Wu Y, Ma W, Zhang S, Zhang YQ. Adoptive T-cell therapy of prostate cancer targeting the cancer stem cell antigen EpCAM. BMC Immunol. 2015;16(1):1.

17. Zheng Z, Chinnasamy N, Morgan RA. Protein L: a novel reagent for the detection of Chimeric Antigen Receptor (CAR) expression by flow cytometry. J Transl Med. 2012;10:29.

18. Macintyre AN, Finlay D, Preston G, Sinclair LV, Waugh CM, Tamas P, et al. Protein kinase $B$ cotrols transcriptional programs that direct cytotoxic $T$ cell fate but is dispensable for T cell metabolism. Immunity. 2011;34:224-36.

19. Wohlfert EA, Clark RB. Vive la resistance!'-the PI3K-Akt pathway can determine target sensitivity to regulatory $T$ cell suppression. Trends Immunol. 2007;28(4):154-60.

20. Sun J, Dotti G, Huye LE, Foster AE, Savoldo B, Gramatges MM, et al. T cells expressing constitutively active Akt resist multiple tumor-associated inhibitory mechanisms. Mol Ther. 2010;18(11):2006-17.

21. Lu B, Wang L, Stehlik C, Medan D, Huang C, Hu S, et al. Phosphatidylinositol 3-kinase/Akt positively regulates Fas (CD95)-mediated apoptosis in epidermal Cl41 cells. J Immunol (Baltimore, Md: 1950). 2006;176(11):6785-93.

22. Bhavsar SK, Merches K, Bobbala D, Lang F. AKT/SGK-sensitive phosphorylation of GSK3 in the regulation of L-selectin and perforin expression as well as activation induced cell death of T-lymphocytes. Biochem Biophys Res Commun. 2012;425(1):6-12.

23. Crompton JG, Sukumar M, Roychoudhuri R, Clever D, Gros A, Eil R, et al. Akt inhibition enhances expansion of potent tumor-specific lymphocytes with memory cell characteristics. Cancer Res. 2015;75(2):296-305.

24. Kim EH, Sullivan JA, Plisch EH, Tejera MM, Jatzek A, Choi KY, et al. Signal integration by Akt regulates CD8 T cell effector and memory differentiation. J Immunol. 2012;188(9):4305-14.

\section{Submit your next manuscript to BioMed Central and take full advantage of:}

- Convenient online submission

- Thorough peer review

- No space constraints or color figure charges

- Immediate publication on acceptance

- Inclusion in PubMed, CAS, Scopus and Google Scholar

- Research which is freely available for redistribution 